\section{Commentary}

\section{RONALD ILLINGWORTH}

\section{Children's Hospital Sheffield}

It is good to be reminded of drugs that not so long ago passed away in disgrace, for someone may have failed to bury a drug or may have mistakenly resurrected it, having forgotten its dangers. Not so many years ago I missed a diagnosis of Pink disease (but was put right by my friend John Black): a chemist had found a large packet of mercurycontaining teething powders.

In the 1940 s and 1950 s there were many articles about the dangers of boric applications to nappy rashes or burns, or of glycerine and borax for oral thrush, or for use with a pacifier-with consequent diarrhoea and vomiting, a red beefy rash, convulsions, meningism, opisthotonos, haemorrhages, circulatory collapse, and death. All my students were shown an impressive colour photograph of an infant dying with a diffuse red beefy rash due to a 'pacifier' dipped in glycerine of borax.

This case report of convulsions arising from the use of glycerine of borax is a timely reminder that when taking a history about a sick child, particularly if the diagnosis is not obvious, one should always ask what medicines or applications, prescribed or not prescribed, have been given.

\title{
Influence of pre-eclampsia on concentrations of haemostatic factors in mothers and infants
}

\author{
J A D SPENCER, M J SMITH, S A CEDERHOLM-WILliAMS, AND A R WILKINSON \\ Departments of Paediatrics and Haematology, John Radcliffe Hospital, Headington, Oxford
}

\begin{abstract}
SUMMARY The effect of pre-eclampsia on selected maternal and neonatal coagulation factors was studied by comparing plasma concentrations in 5 severely pre-eclamptic and 5 normal pregnancies. Pre-eclampsia was associated with appreciably lower maternal values of antithrombin III, plasminogen, and platelet count in late pregnancy but umbilical cord blood values showed no notable differences from normal pregnancy.
\end{abstract}

Pre-eclampsia is associated with an exaggeration of the haemostatic changes of normal pregnancy to the extent that intravascular coagulation may be shown. ${ }^{1}$ Only 2 studies have reported haemostatic factors in the newborn after pre-eclamptic pregnancy. Hathaway et al. found a raised umbilical cord concentration of factor VIII and the concentration of antithrombin III was half that of nonpregnant adults. ${ }^{2}$ Condie showed no important differences in umbilical cord blood values of fibrinogen, factor $X$, plasminogen, $\alpha_{2}$ macroglobulin, and $\alpha_{1}$ antitrypsin but found that fibrinolytic activity was appreciably lower in the cord blood of the pre-eclamptic group ${ }^{3}$ suggesting the possibility of a predisposition to intravascular coagulation in these neonates at birth. In this study of mothers in late pregnancy and their term infants, plasma concentrations of selected haemostatic factors in normal and pre-eclamptic pregnancies are compared.

\section{Patients and methods}

Five women with severe pre-eclampsia and 5 women with uncomplicated singleton pregnancies were matched for parity and mode of delivery. Maternal venous blood samples were collected before induction of labour or elective caesarean section. Umbilical cord venous blood was collected after the cord was clamped and divided but before delivery of the placenta. A free flowing venous blood sample was collected from each neonate on the 6th day of life at the time of routine screening for phenylketonuria and hypothyroidism.

Total plasma concentrations of fibrinogen, prothrombin, factor VIII, factor X, antithrombin III, plasminogen, $\alpha_{2}$ antiplasmin, $\alpha_{2}$ macroglobulin, and $\alpha_{1}$ antitrypsin were estimated by radial immunodiffusion and enzymatic assay using low molecular weight chromogenic substrates adapted for very small samples. ${ }^{4}$ The cord and neonatal results were corrected to a haematocrit value of $45 \%$. The study was approved by the hospital ethical committee and informed consent was obtained from all the mothers. 